MODELING, IDENTIFICATION AND CONTROL, 1990, vOL. 11, NO. 3, 127-140

doi:10.4173/mic.1990.3.1

\title{
Rational transfer function approximations to transport delay
}

\author{
JENS G. BALCHEN $\dagger$
}

Keywords: Transport delay approximation, Padé approximation.

A method is presented for determining a rational transfer function approximation to transport delay which has the property that the error in the frequency response phase shift is zero at certain frequencies. This new approximation is particularly suitable for the determination of stability conditions for feedback systems containing transport delay.

\section{Introduction}

A familiar problem is finding means of approximating the transfer function of a transport delay (pure delay) with a rational transfer function. Such approximations may be introduced to derive conditions for the stability of feedback systems based upon algebraic stability criteria. A second application is deriving state space model approximations for systems with delay. This note considers different rational approximations to the transfer function $\mathrm{h}(\mathrm{s})=\boldsymbol{e}^{-\tau s}$. These are often referred to as Padéapproximations (Vlach 1969, Luke 1975).

\section{Alternative approximations}

A common way of approaching the approximation problem is to express the original transfer function as follows:

$$
e^{-\tau s}=\frac{e^{-\tau / 2 s}}{e^{+\tau / 2 s}}
$$

Thereby it is possible to arrive at balanced approximations where the complex function vector length stays the same, namely 1 during the approximation.

When the numerator and the denominator terms of (1) are expanded in a power series, the following approximation is obtained:

$$
\begin{gathered}
e^{-\tau s} \approx \frac{1-\frac{\tau}{2} s+\frac{1}{2}\left(\frac{\tau}{2}\right)^{2} s^{2}-\frac{1}{6}\left(\frac{\tau}{2}\right)^{3} s^{3}+\ldots}{1+\frac{\tau}{2} s+\frac{1}{2}\left(\frac{\tau}{2}\right)^{2} s^{2}+\frac{1}{6}\left(\frac{\tau}{2}\right)^{3} s^{3}+\ldots} \\
=\frac{1-\frac{\tau}{2} s+\frac{\tau^{2}}{8} s^{2}-\frac{\tau^{2}}{48} s^{3}+\ldots}{1+\frac{\tau}{2} s+\frac{\tau^{2}}{8} s^{2}+\frac{\tau^{3}}{48} s^{3}+\ldots}=\frac{1-a_{1} s+a_{2} s^{2}-a_{3} s^{3}+\ldots}{1+a_{1} s+a_{2} s^{2}+a_{3} s^{3}+\ldots}
\end{gathered}
$$

Received 20 April 1990.

$\dagger$ Division of Engineering Cybernetics, The Norwegian Institute of Technology, 7034 Trondheim, Norway. 
This approximation will be referred to as 'approximation $A_{n}$ ' where $n$ is the order. It is usually assumed that the accuracy of the approximation is improved by increasing the number of terms in (2). However as will be shown below, this is not necessarily so.

Another common approximation has the form

$$
\begin{aligned}
e^{-\tau s} \approx & \frac{\left(1-\frac{\tau}{2 n} s\right)^{n}}{\left(1+\frac{\tau}{2 n} s\right)} \\
= & \frac{1-n\left(\frac{\tau}{2 n} s\right)+\frac{n(n-1)}{2 !}\left(\frac{\tau}{2 n} s\right)^{2}-\frac{n(n-1)(n-2)}{3 !}\left(\frac{\tau}{2 n} s\right)^{3}+\ldots}{1+n\left(\frac{\tau}{2 n} s\right)+\frac{n(n-1)}{2 !}\left(\frac{\tau}{2 n} s\right)^{2}+\frac{n(n-1)(n-2)}{3 !}\left(\frac{\tau}{2 n} s\right)^{3}+\ldots} \\
= & \frac{1-b_{1} s+b_{2} s^{2}-b_{3} s^{3}+\ldots}{1+b_{1} s+b_{2} s^{2}+b_{3} s^{3}+\ldots} \\
= & \left.\frac{1-\frac{\tau}{2} s+\frac{\tau^{2}}{12} s^{2}-\frac{\tau^{3}}{216} s^{3}}{1+\frac{\tau}{2} s+\frac{\tau^{2}}{12} s^{2}+\frac{\tau^{3}}{216} s^{3}} \text { (for } n=3\right)
\end{aligned}
$$

This is 'approximation $B_{n}$ '.

The problem with these approximations is that they do not seem to be the best possible when the power series are truncated to the second or third orders for instance.

The purpose of the present investigation is to find an approximation of the form

$$
e^{-\tau s}=\frac{1-c_{1} s+c_{2} s^{2}-c_{3} s^{3}+\ldots}{1+c_{1} s+c_{2} s^{2}+c_{3} s^{3}+\ldots}
$$

in which $c_{1}, c_{2}, c_{3} \ldots$ are to be determined so that the approximation meets certain specifications. This can be done in a number of different ways where the success is dependent upon the application of the results. We shall assume that the result will be used primarily for stability analysis of feedback systems. Therefore it makes sense to look at the frequency response of the transfer function and its approximation, namely

$$
\begin{aligned}
e^{-j \tau \omega} & \approx \frac{1-j c_{1} \omega-c_{2} \omega^{2}+j c_{3} \omega^{3}-\ldots}{1+j c_{1} \omega-c_{2} \omega^{2}-j c_{3} \omega^{3}+\ldots} \\
& =\frac{1-c_{2} \omega^{2}-j\left(c_{1} \omega-c_{3} \omega^{3}\right)-\ldots}{1-c_{3} \omega^{2}+j\left(c_{1} \omega-c_{3} \omega^{3}\right)+\ldots}
\end{aligned}
$$

It is observed that the real and imaginary terms appearing in the numerator and the denominator of (5) have the same magnitude. This is a property of the balanced approximation.

\section{Approximating the frequency response}

Since the magnitude of the complex vector of (5) is unity for all frequencies $\omega$, the approximation must be determined from the phase angle. 
The phase angle of the exact frequency response of a delay is

$$
\angle e^{-j \tau \omega}=-\tau \omega
$$

whereas the approximation of (5) yields the phase angle

$$
\angle e^{-j r \omega} \approx-2 \arctan \left(\frac{c_{1} \omega-c_{3} \omega^{3}}{1-c_{2} \omega^{2}}\right) \quad \text { for } n=3
$$

One way of determining the unknown coefficients of (5) would be to minimize the integral of the squared error between (6) and (7) over a certain frequency range. We shall choose to determine the unknown coefficients by prescribing that the approximation (7) is to fit the exact expression (6) at certain angles namely $-\pi / 2,-\pi,-3 \pi / 2$. Any other set of angles could have been chosen but these are advantageous because they play important roles in the analysis of many control systems. This will be referred to as 'approximation $C_{n}$ '.

When the approximation of (5) is of the first order $\left(c_{2}=c_{3}=0\right)$, the maximal negative phase angle will be $-\pi$ and we are free to choose one phase angle where the approximation is to fit the exact value. Choosing this to be $-\pi / 2$, we obtain the coefficient of the first order approximation in (7) through

$$
-2 \arctan c_{1} \omega=-\tau \omega=-\frac{\pi}{2}
$$

giving

$$
\omega_{1}=\frac{\pi}{2 \tau}
$$

and

$$
-\arctan c_{1} \omega=-\frac{\pi}{4}
$$

This leads to

$$
c_{1} \omega=1
$$

or

$$
c_{1}=\frac{2}{\pi} \tau
$$

Thus the first order approximation which has the property of giving the correct phase angle at $-\pi / 2$ will be

$$
C_{1}: e^{-\tau s} \approx \frac{1-\frac{2}{\pi} \tau s}{1+\frac{2}{\pi} \tau s}
$$

This can be compared with the approximations $A_{1}$ of (2) and $B_{1}$ of (3) and it is seen that the coefficient $\left(c_{1}\right)$ has changed from $0.5 \tau$ to $0.637 \tau$.

Evaluating $C_{2}$ (the second order C approximation), we can make (6) and (7) equal at two frequencies, namely $\omega_{1}=\pi / 2 \tau$ and $\omega_{2}=\pi / 2$. At these frequencies the phase angles are $-\pi / 2$ and $-\pi$ respectively. 
Applying (7) yields

$$
\begin{aligned}
& \frac{c_{1} \omega_{1}}{1-c_{2} \omega_{1}^{2}}=\frac{\pi}{4}=1 \\
& \frac{c_{1} \omega_{2}}{1-c_{2} \omega_{2}^{2}}=\tan \frac{\pi}{2}=\infty
\end{aligned}
$$

(13) yields

$$
c_{2}=\left(\frac{1}{\omega_{2}}\right)^{2}=\left(\frac{\tau}{\pi}\right)^{2}
$$

which when applied in (12) leads to

$$
c_{1}=\frac{1}{\omega_{1}}-c_{2} \omega_{1}=\frac{3 \tau}{2 \pi}
$$

Thus the second order approximation with the property that the phase angle is exact at $-\pi / 2$ and $-\pi$ will be

$$
C_{2}: e^{-\tau s} \approx \frac{1-\frac{3 \tau}{2 \pi} s+\left(\frac{\tau}{\pi}\right)^{2} s^{2}}{1+\frac{3 \tau}{2 \pi} s+\left(\frac{\tau}{\pi}\right)^{2} s^{2}}
$$

Comparing the result of (16) with the approximations $A_{2}$ of (2) and $B_{2}$ of (3) we see that the first order coefficient $\left(c_{1}\right)$ has changed from $0.5 \tau$ to $0.477 \tau$ and that the second order coefficient $\left(c_{2}\right)$ has changed from $0.125 \tau^{2}$ in approximation $A_{2}$ and $0.0833 \tau^{2}$ in $B_{2}$ to $0 \cdot 101 \tau^{2}$ in $A_{2}$. In other words, the second order coefficient $\left(c_{2}\right)$ in $C_{2}$ lies between those of $A_{2}$ and $B_{2}$.

Proceeding to the third order approximation, we find from (6) and (7)

$$
\begin{aligned}
& \frac{c_{1} \omega_{1}-c_{3} \omega_{1}^{3}}{1-c_{2} \omega_{1}^{2}}=\tan \frac{\pi}{4}=1 \\
& \frac{c_{1} \omega_{2}-c_{3} \omega_{2}^{3}}{1-c_{2} \omega_{2}^{2}}=\tan \frac{\pi}{2}=\infty \\
& \frac{c_{1} \omega_{3}-c_{3} \omega_{3}^{3}}{1-c_{2} \omega_{3}^{2}}=\tan \frac{3 \pi}{4}=-1
\end{aligned}
$$

where

$$
\omega_{1}=\frac{\pi}{2 \tau}, \quad \omega_{2}=\frac{\pi}{\tau} \quad \text { and } \quad \omega_{3}=\frac{3 \pi}{2 \tau} .
$$

(18) yields

$$
c_{2}=\left(\frac{\tau}{\pi}\right)^{2}=\frac{\tau^{2}}{9 \cdot 87}
$$

which when applied to (17) and (19) leads to

$$
c_{1}=\frac{\tau}{\frac{12}{19} \pi}=\frac{\tau}{1.984}
$$


and

$$
c_{3}=\frac{1}{3}\left(\frac{\tau}{\pi}\right)^{3}=\frac{\tau^{2}}{93 \cdot 02}
$$

Consequently, the third order approximation which offers exact phase shift at $-\pi / 2,-\pi$ and $-3 \pi / 2$ will be

$$
\begin{array}{r}
C_{3}: e^{-\tau s} \approx \frac{1-\frac{\tau}{12} s+\frac{\tau^{2}}{\pi^{2}} s^{2}-\frac{\tau^{3}}{3 \pi^{3}} s^{3}}{1+\frac{\tau}{\frac{12}{19} \pi} s+\frac{\tau^{2}}{\pi^{2}} s+\frac{\tau^{3}}{3 \pi^{3}} s^{3}} \\
=\frac{1-\frac{\tau}{1.984} s+\frac{\tau^{2}}{9 \cdot 87} s^{2}-\frac{\tau^{3}}{93.02} s^{3}}{1+\frac{\tau}{1.984} s+\frac{\tau^{2}}{9.87} s^{2}+\frac{\tau^{3}}{93.02} s^{3}}
\end{array}
$$

The latter expression can be compared with (2) and (3) and it is seen that the second and third order coefficients in particular are quite different.

A general formula for determining coefficients $c_{1}, c_{2}, c_{3} \ldots$ can be developed. Expressing these unknown coefficients as a vector

$$
c=\left[c_{1} c_{2} c_{3} \ldots c_{n}\right]^{\mathrm{T}}
$$

the conditions of (17)-(19) can in general be expressed as

$$
\Omega_{n} c=p
$$

where $\Omega_{n}$ for the case of $n=5$ has the form

where

$$
\Omega_{5}=\left[\begin{array}{ccccc}
\omega_{1} & \omega_{1}^{2} & -\omega_{1}^{3} & -\omega_{1}^{4} & -\omega_{1}^{5} \\
0 & \omega_{2}^{2} & 0 & -\omega_{2}^{4} & 0 \\
\omega_{3} & -\omega_{3}^{2} & -\omega_{3}^{3} & \omega_{3}^{4} & -\omega_{3}^{5} \\
\omega_{4} & 0 & -\omega_{4}^{3} & 0 & -\omega_{4}^{5} \\
\omega_{5} & \omega_{5}^{2} & -\omega_{5}^{3} & -\omega_{5}^{4} & -\omega_{5}^{5}
\end{array}\right]
$$

$$
\omega_{n}=n \frac{\pi}{2}
$$

Furthermore $p$ for the case $n=5$ in (24) is

$$
\boldsymbol{p}=\left[\begin{array}{lllll}
1 & 1 & -1 & 0 & 1
\end{array}\right]^{\mathrm{T}}
$$

The solution of (24) is

$$
c=\Omega_{n}^{-1} p
$$

The results of (11), (16) and (23) are easily verified using (27) with $n=1,2$ and 3 respectively. 


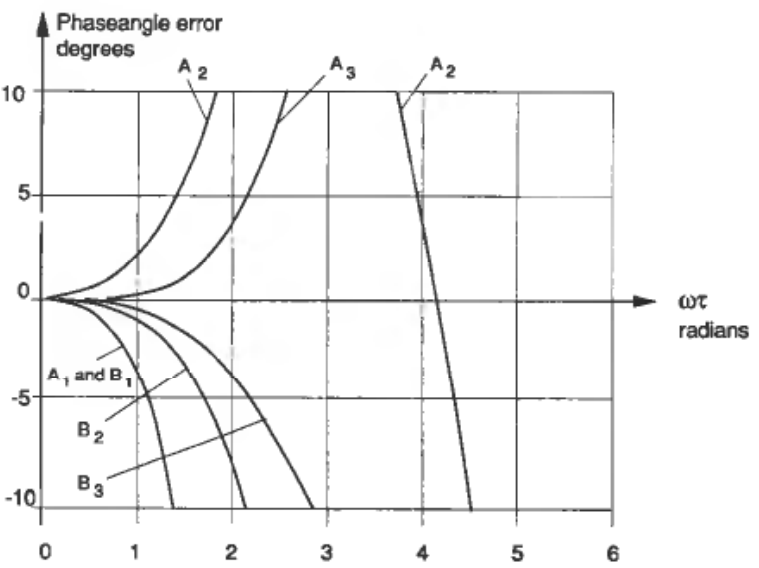

(b)

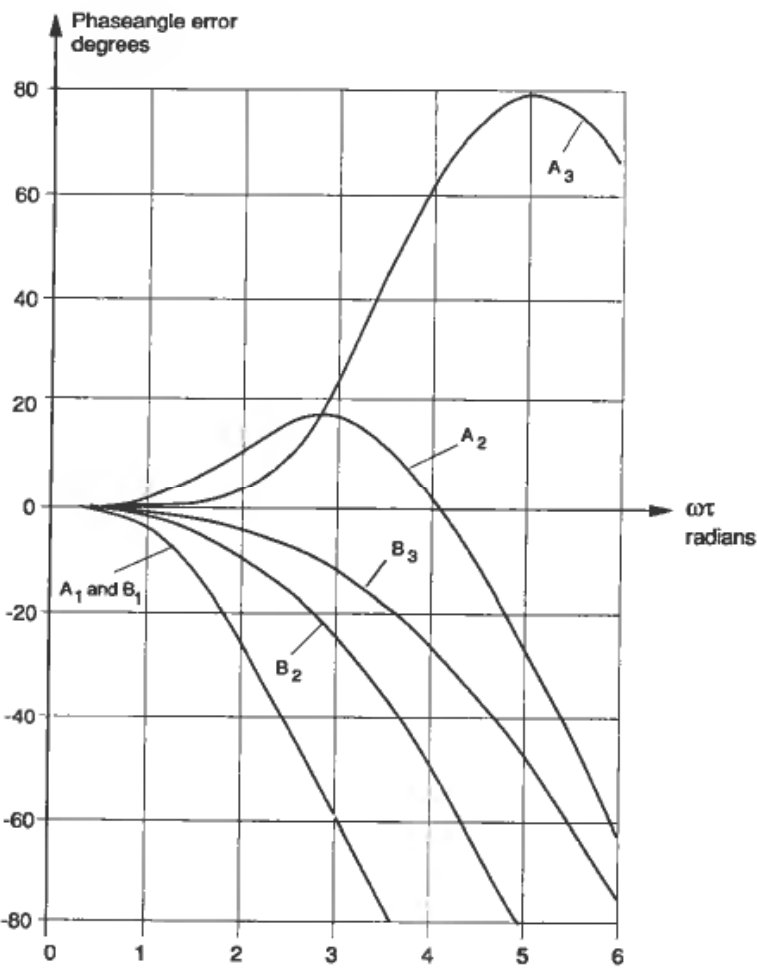

(c)

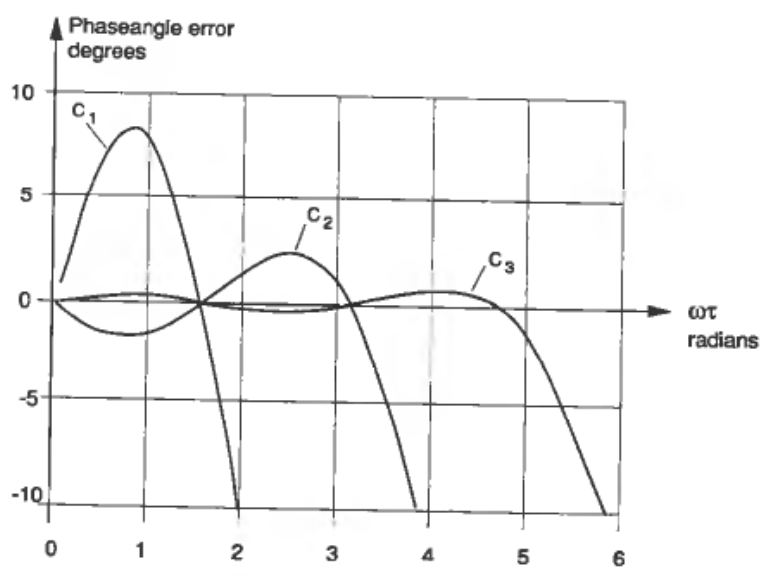

Figure 1. The phase angle error of the approximations $A_{n}, B_{n}$ and $C_{n}(a) A_{n}$ and $B_{n}$ at low frequencies. (b) $A_{n}$ and $B_{n}$ at higher frequencies. (c) $C_{n}$ - 
The errors associated with each of the three approximations have been investigated and are shown graphically in Fig. 1. It is clearly demonstrated that the new type of approximation $\left(C_{n}\right)$ is superior to that of $\left(A_{n}\right)$ and $\left(B_{n}\right)$. The maximal errors indicated in the figure are quite small.

In some applications it is interesting to compare the pole-zero-plot of each of the approximations. Such plots are shown in Fig. 2 for the three different approximations studied above. It is observed that the $A_{5}$ and $A_{6}$ approximations have two poles in the right half of the $s$-plane. This means that the approximation is an unstable system and cannot be used alone. But these poles will be forced towards the left in a feedback system and thereby still make an acceptable approximation.

A third way of comparing the different approximations, is by simulating their time responses. This can be done by deriving the equivalent state space models for each of the rational transfer functions.

Two different input time functions have been applied for test purposes. The first input function is a step function (Laplace transform $u_{1}(s)=1 / s$ ) and the second is a 'soft' step function (Laplace transform $u_{2}(s)=1 / s(1+\tau s)$, i.e. with a time constant equal to the time delay $\tau$.

The results are shown in Figs. 3-7. Figure 3 shows the step response of approximation $A_{n}$ for $n=1,2 \ldots 5$. As can be seen, the approximation $A_{5}$ is unstable as indicated above. It can hardly be said that the approximation improves with increasing $n$. Figure 4 shows the responses to a 'soft' step of approximations $A_{n}$. These appear to be much more successful than the pure step response. The reason is that a soft step contains less energy at high frequencies.

Figure 5 shows the step response and Fig. 6 the 'soft' step response of approximations $B_{n}$. In this case it is fair to say that the success of the approximation improves with increasing $n$. This is particularly clear for the 'soft' step response in Fig. 6.

Figures 7 and 8 show the responses of approximations $C_{n}$ to step inputs and 'soft' step inputs respectively. Again it is observed that the approximation improves with increasing $n$.

Figures $9(a)$ and $9(b)$ compares the 'soft' step response of the three approximations with $n=2$ and $n=3$ respectively. It is evident that approximation $C$ comes out best even though the differences between the three are not very significant.

A conclusion to be drawn from the above investigations is that the approximation $C_{3}$ (as given in (23)), is very successful and should be used where high accuracy is required. Approximation $C_{2}$ is the seond best and will probably be acceptable in most cases even for stability investigations.

\section{Applications}

The rational function approximation to the transfer function of delay can be used for a number of purposes (Balchen 1977). It is immediately observed with algebraic expressions of conditions for stability of feedback systems of the form shown in Fig. 10 that using the approximations $C_{1}, C_{2}$ and $C_{3}$ the result will be exact if the phase angle of $h(j \omega)$ is $-\pi / 2$. Such is the case if

$$
h(s)=\frac{K}{s}
$$

and also for other transfer functions under certain conditions. The approximations of $C_{2}$ and $C_{3}$ will yield the exact answer if the phase angle of $h(j \omega)$ is 0 which will occur if 


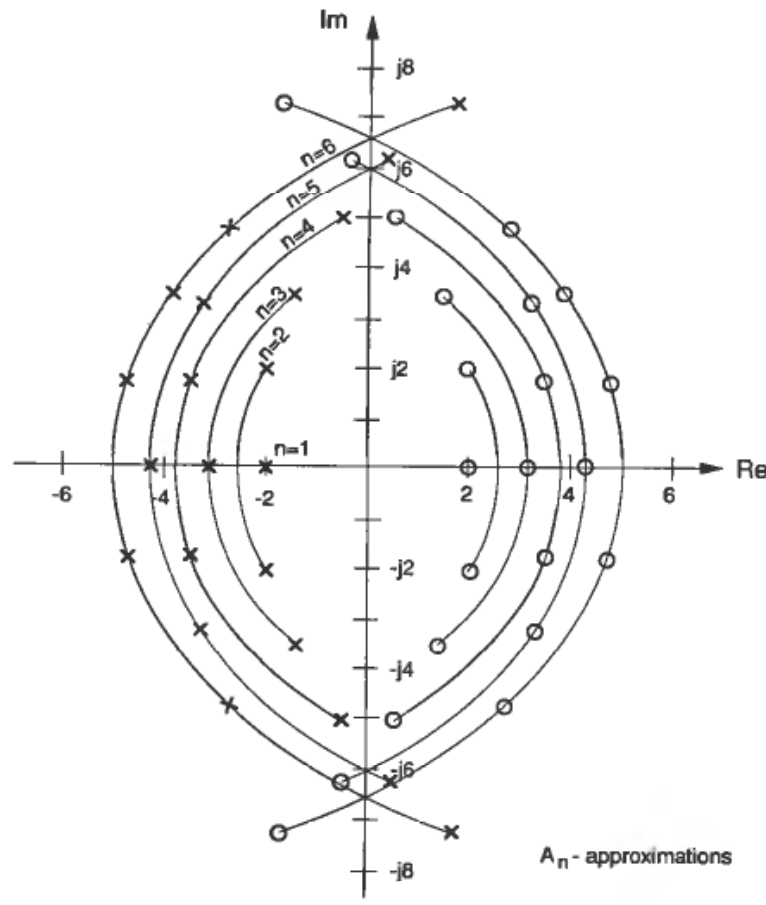

(a)

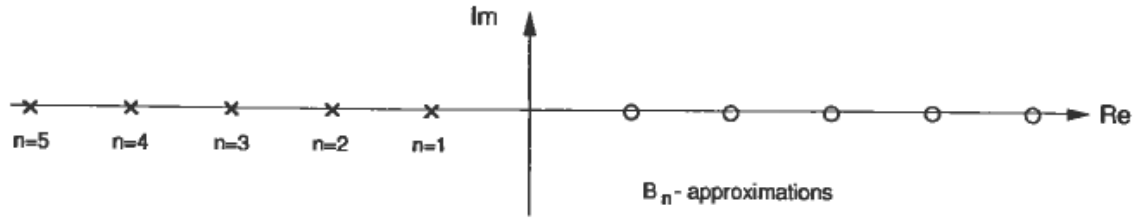

(b)

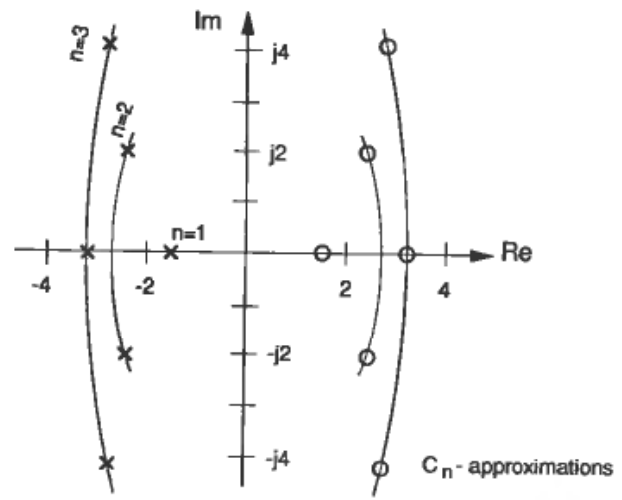

(c)

Figure 2. Pole-zero-configuration of different approximations. (a) $A_{n}$-approximations. (b) $B_{n}$-approximations. (c) $C_{n}$-approximations. 

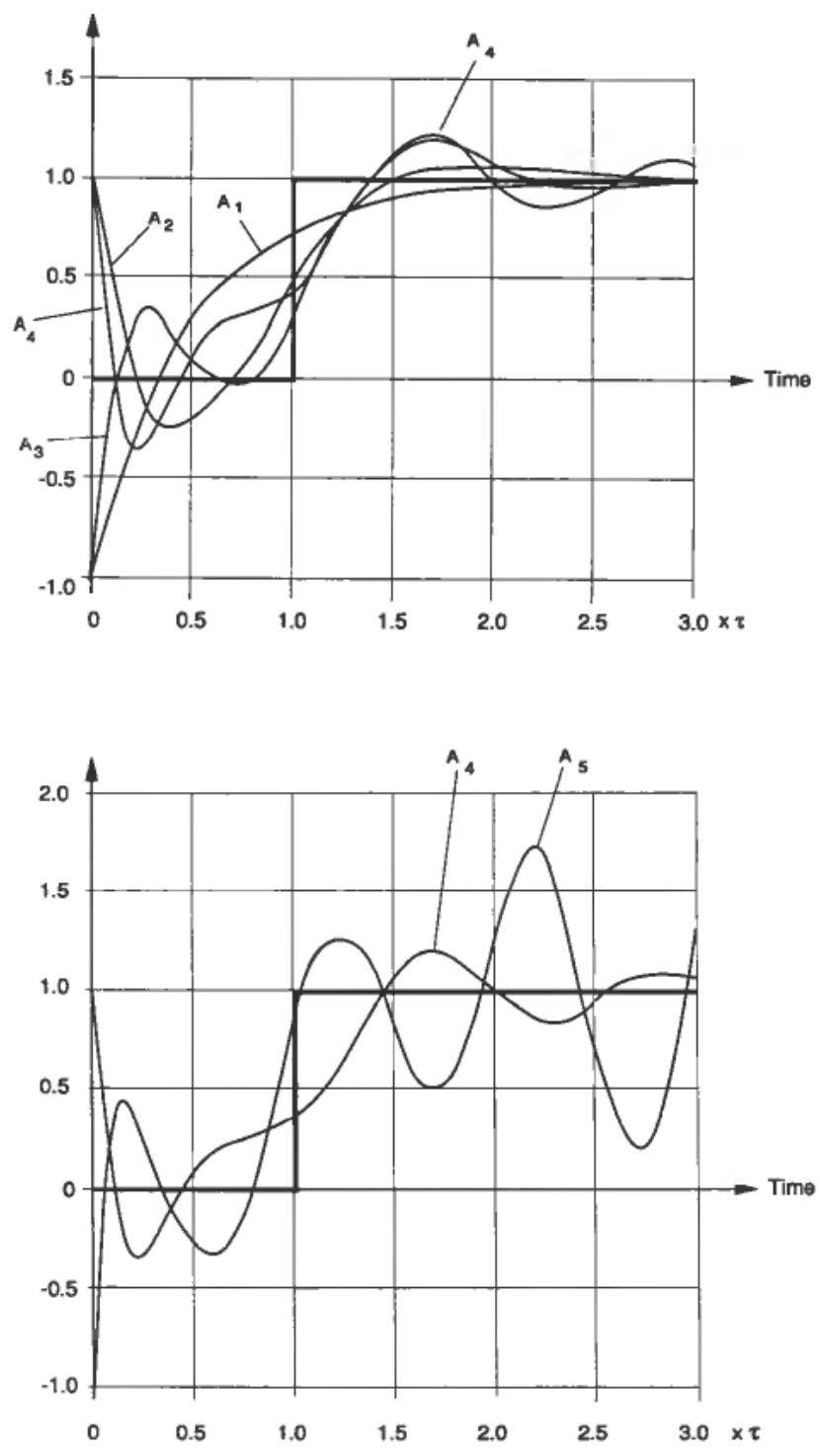

Figure 3. Step response of $A_{n}$-approximations. 

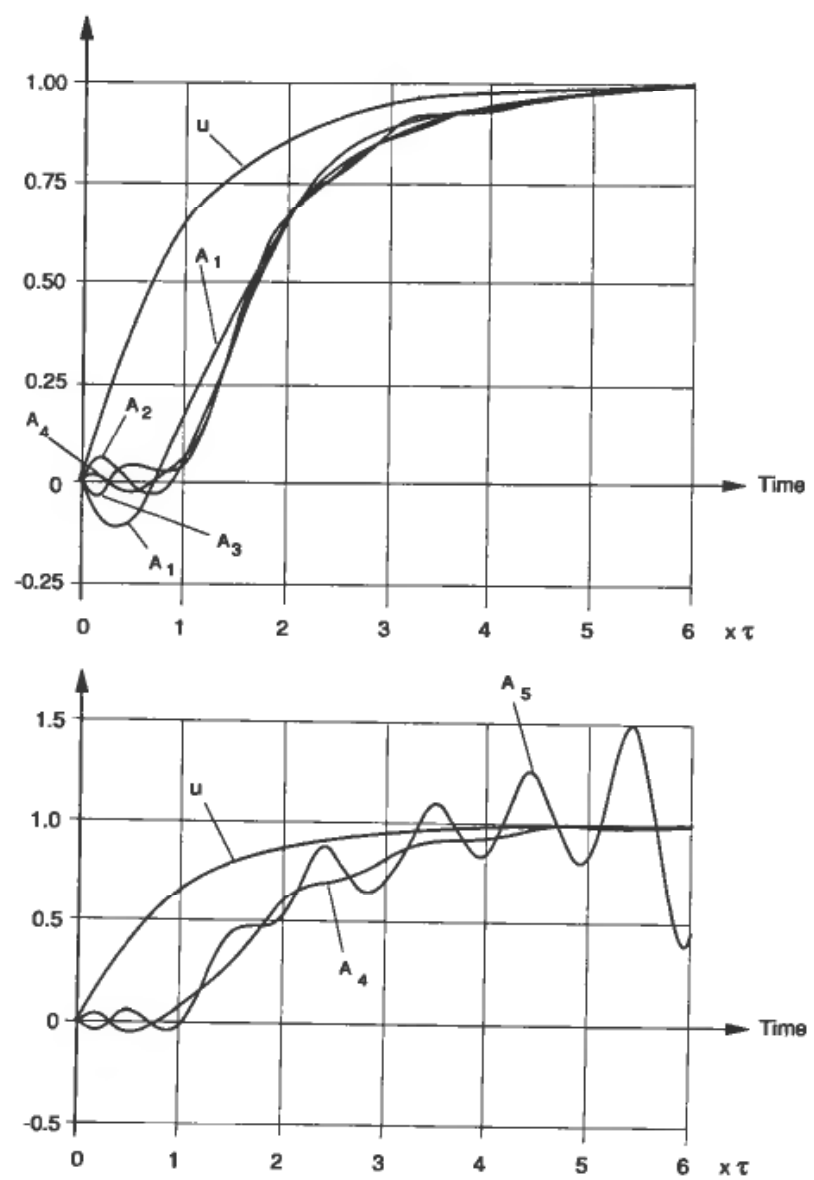

Figure 4. 'Soft' step response of $A_{n}$-approximations.

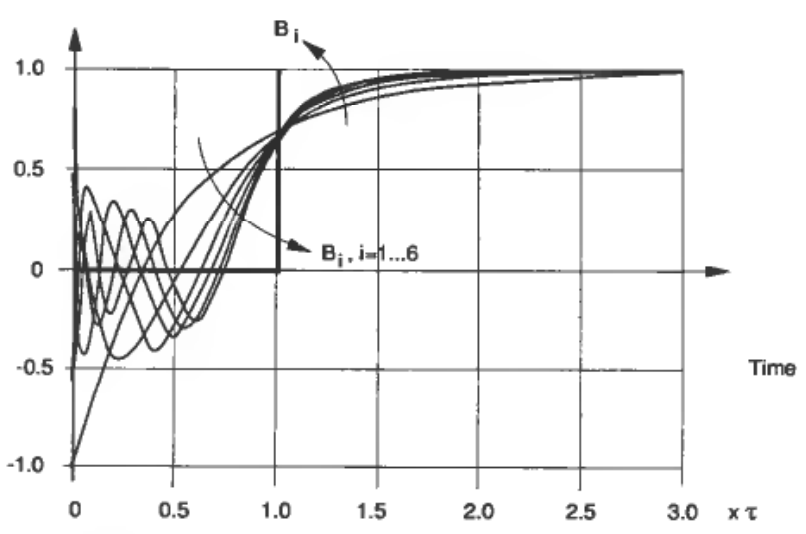

Figure 5. Step response of $B_{n}$-approximations. 


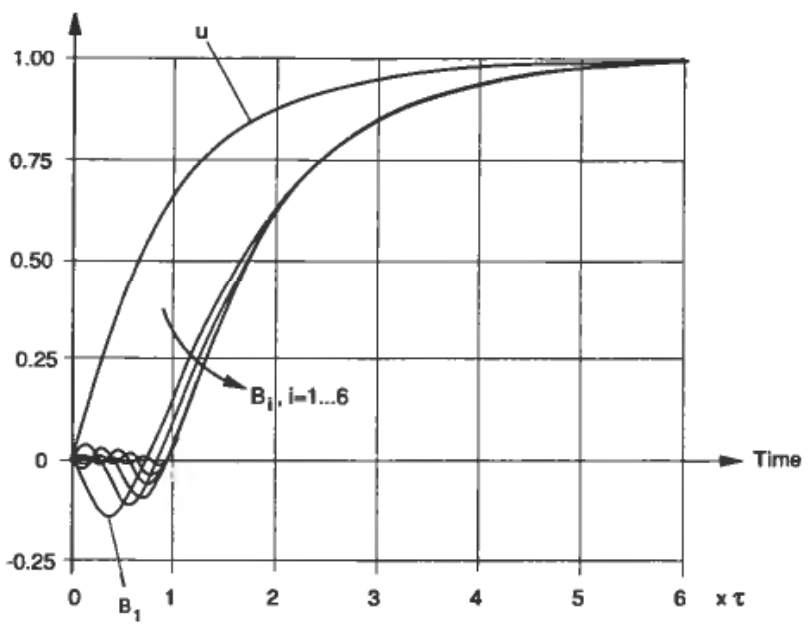

Figure 6. 'Soft' step response of $B_{n}$-approximations.

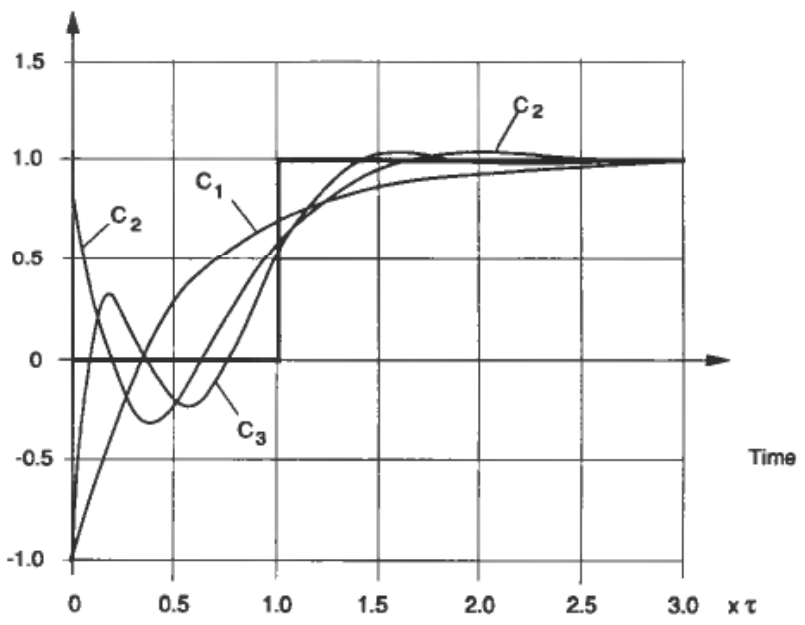

Figure 7. Step response of $C_{n}$-approximations.

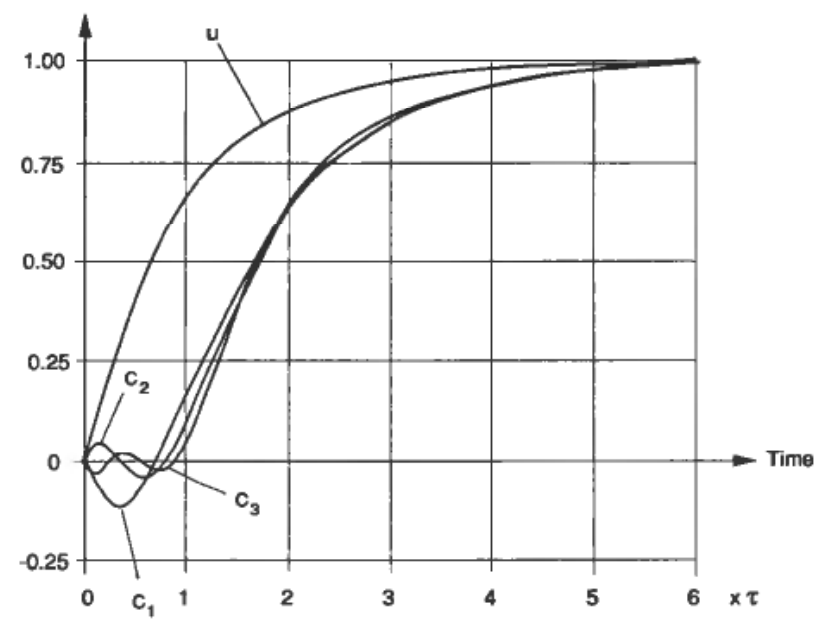

Figure 8. 'Soft' step response of $C_{n}$-approximations. 
138

J. G. Balchen

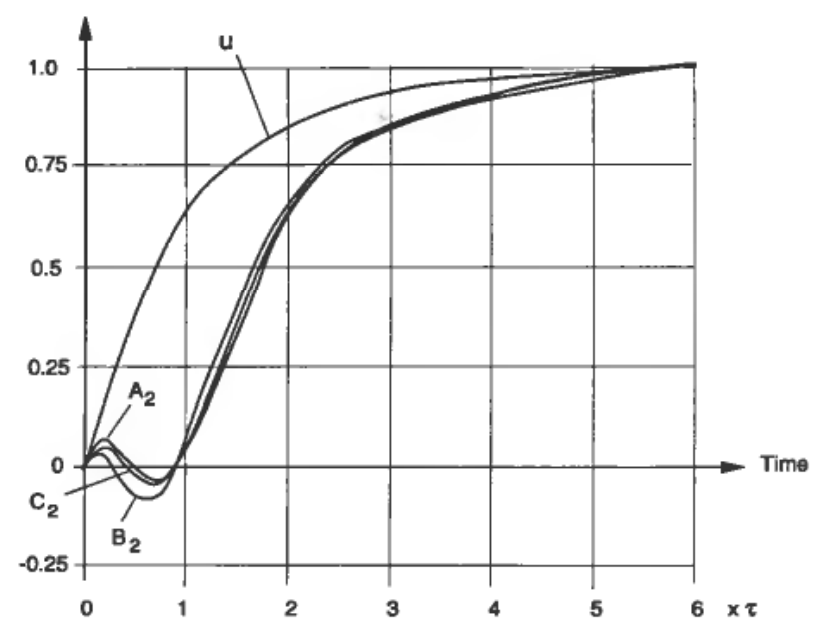

(a)

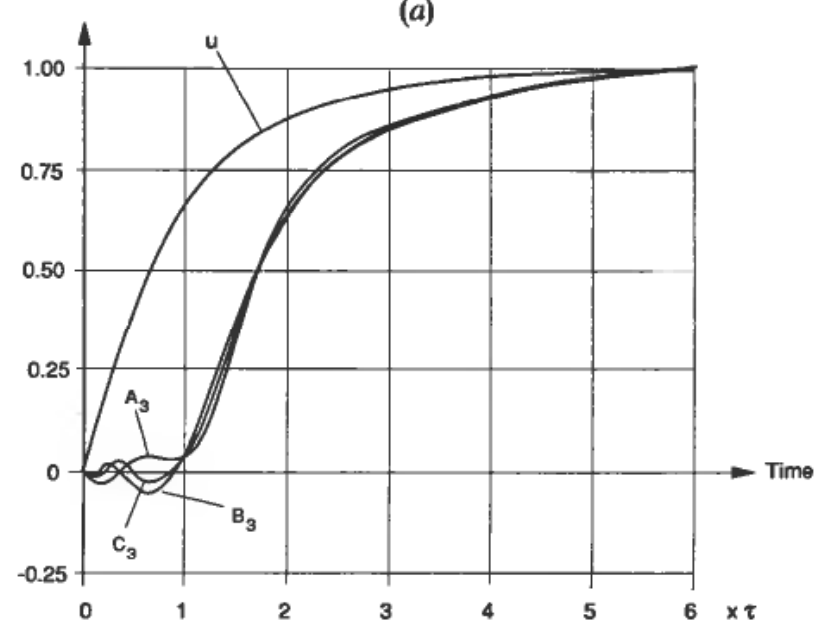

(b)

Figure 9. Comparison of 'soft' step responses. (a) $A_{2}, B_{2}, C_{2}$-approximations. (b) $A_{3}, B_{3}$, $C_{3}$-approximations.

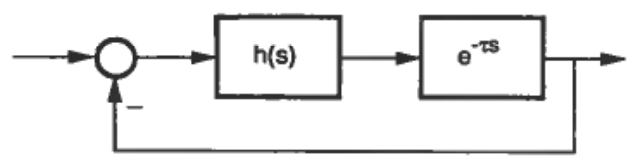

Figure 10. Feedback control system involving transport delay.

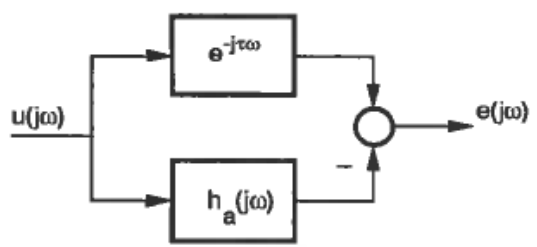

Figure 11. Generating the error between the transport delay and its approximation in the frequency domain. 
$h(s)=K$ and for other transfer functions under certain conditions. Since the error in the phase angle is very small for the approximations $C_{2}$ and $C_{3}$, conditions for stability for any feedback system will be determined with quite high accuracy using these two approximations.

The error in predicting the response in the time domain when using the approximation in the frequency domain can be studied by a number of techniques. One of these is based upon Parseval's theorem which states

$$
\int_{0}^{\infty} e^{2}(t) d t=\frac{1}{2 \pi j} \int_{-j \infty}^{+j \infty} e(s) e(-s) \mathrm{d} s=\frac{1}{\pi} \int_{0}^{\infty}|e(j \omega)|^{2} d \omega
$$

In other words, the integral of the square error in the time domain is equal to the integral of the square error in the frequency domain. The latter is easily derived for the above approximations $\left(h_{a}(j \omega)\right)$ as illustrated in Fig. 11.

$$
\begin{aligned}
e(j \omega) & =\left(e^{-j \tau \omega}-h_{a}(j \omega)\right) u(j \omega) \\
& =e^{-j \tau \omega}\left(1-e^{j\left(L h_{a}+\tau \omega\right)}\right) u(j \omega) \\
& =e^{-j \tau \omega}(1-(\cos \Delta \varphi(\omega)+j \sin \Delta \varphi(\omega))) u(j \omega)
\end{aligned}
$$

Applying this to (29) we get

$$
\begin{aligned}
\int_{0}^{\infty} e^{2}(t) d t & =\frac{1}{\pi} \int_{0}^{\infty} 2\left(1-\cos \Delta \varphi(\omega)|u(j \omega)|^{2} d \omega\right. \\
& =\frac{4}{\pi} \int_{0}^{\infty}\left(\sin \frac{1}{2} \Delta \varphi(\omega)\right)^{2}|u(j \omega)|^{2} d \omega
\end{aligned}
$$

The last term under the integral for a step function and a 'soft' step function respectively will be

$$
|u(j \omega)|^{2}=\frac{1}{\omega^{2}}
$$

and

$$
|u(j \omega)|^{2}=\frac{1}{\omega^{2}\left(1+\tau^{2} \omega^{2}\right)}
$$

Using (7) it follows that

$$
\frac{1}{2} \Delta \varphi(\omega)=-\frac{\tau \omega}{2}+\arctan \quad \omega c_{1} \frac{1-\frac{c_{3}}{c_{1}} \omega^{2}}{1-c_{2} \omega^{2}}
$$

for approximation $C_{3}$ and similarly for approximations $A_{3}$ and $B_{3}$. When $\omega \rightarrow 0$, we see that

$$
\frac{1}{2} \Delta \varphi(\omega) \approx-\omega\left(\frac{\tau}{2}-c_{1}\right)
$$

which when applied to (31) shows that the integrand will approach $\left(\tau / 2-c_{1}\right)^{2}$ when $\omega \rightarrow 0$. The similar expression for approximations $A_{3}$ and $B_{3}$ will be 0 since $a_{1}=b_{1}=\tau / 2$. Thus the contribution to the integral of (31) will be slightly higher at the lowest frequencies for approximation $C_{3}$, but at higher frequencies the contribution to the integral will be smaller using approximation $C_{3}$ rather than $A_{3}$ or $B_{3}$. 


\section{Conclusions}

A new rational transfer function approximation for transport delay has been developed and compared with the approximations most commonly used in the literature. It has been shown that the new approximation is superior particularly when applied to the determination of conditions for stability in feedback control systems containing elements with delay.

\section{Acknowledgment}

The author acknowledges the assistance of Mr David di Ruscio who did some of the numerical calculations appearing in this paper.

\section{REFERENCES}

BALCHEN, J. G. (1977). Control Engineering, Vol. 1 (Tapir Book Company, Trondheim, Norway) in Norwegian.

LUKE, Y. L. (1975). Mathematical Functions and their Approximations (Academic Press, Inc., New York).

VLACH, J. (1969). Computerized Approximation and Synthesis of Networks (John Wiley \& Sons, Inc., New York). 